Check for updates

Cite this: RSC Adv., 2019, 9, 4507

\title{
High-throughput screening of high lactic acid- producing Bacillus coagulans by droplet microfluidic based flow cytometry with fluorescence activated cell sorting $\dagger$
}

\author{
Xu-Dong Zhu, ${ }^{a}$ Xiang Shi, ${ }^{a}$ Shu-Wen Wang, ${ }^{b}$ Ju Chu, ${ }^{a}$ Wei-Hong Zhu, (DD ${ }^{\mathrm{b}}$ \\ Bang-Ce Ye, (D) a Peng Zuo*a and Yong-Hong Wang ${ }^{\star a}$
}

A high-throughput screening system based on droplet microfluidic sorting was developed and employed for screening of high lactic acid-producing Bacillus coagulans. In this system, water-in-oil-in-water (W/O/W) droplets, which were $\sim 12 \mathrm{pL}$ in volume were used as picoliter-reactors for lactic acid fermentation. A fluorescent sensor was developed and used for monitoring $\mathrm{pH}$ which indicated the production of lactic acid. After fermentation, fluorescence activated cell sorting was performed with high sensitivity and speed. Using this microfluidic high-throughput screening system, we found a mutant with a yield of $76 \mathrm{~g} \mathrm{~L}^{-1}$ lactic acid which was $52 \%$ higher than its parent strain with a screening throughput exceeding $10^{6}$ clones per $\mathrm{h}$.

Received 25th November 2018 Accepted 4th January 2019

DOI: $10.1039 / \mathrm{c} 8 \mathrm{ra09684h}$

rsc.li/rsc-advances liquid workstations ${ }^{8-10}$ costs hundreds of thousands of dollars and takes up too much room. ${ }^{11-13}$

Recently, microfluidic technology in combination with fluorescence activated cell sorting (FACS) techniques has brought about an idea to address these issues. ${ }^{14}$ Actually, screening of mutants in monodisperse picoliter compartments, generated at kilohertz speeds in microfluidic devices, is coming of age. ${ }^{15-17}$ After a decade of proof-of-principle experiments, workflows have emerged that combine existing microfluidic modules to assay reaction progress quantitatively and yield improved strains or enzymes. ${ }^{18-23}$ Recent examples of the screening of different kinds of libraries suggest that this approach is not only faster and cheaper, but solves problems beyond the feasibility scope of current methodologies. ${ }^{24-26}$

Herein, we described a high throughput screening system for lactic acid-producing bacteria by which cells are encapsulated in a droplet (water-oil-water). A fluorescent reporter was used to determine $\mathrm{pH}$ changes in the droplet. Droplets were then sorted using flow cytometry based on fluorescence. Compared to the state-of-the-art findings in the reviews suggested above, there was no need for cell lysis to access the phenotype in our system. Besides, double-layer droplets which act as a vessel to FACS made our system highly flexible. Once a correlation between phenotypes and fluorescence intensity was established, this screening could be performed in this system.

\section{Results and discussion}

edu.cn

${ }^{b}$ Institute of Fine Chemicals, East China University of Science and Technology, Shanghai, 200237, China

$\dagger$ Electronic supplementary information (ESI) available. See DOI: 10.1039/c8ra09684h
Here, we built an ultrahigh throughput screening system for high lactic acid-producing Bacillus coagulans using droplet microfluidics and FACS. The flow chart was shown in Fig. 1. 


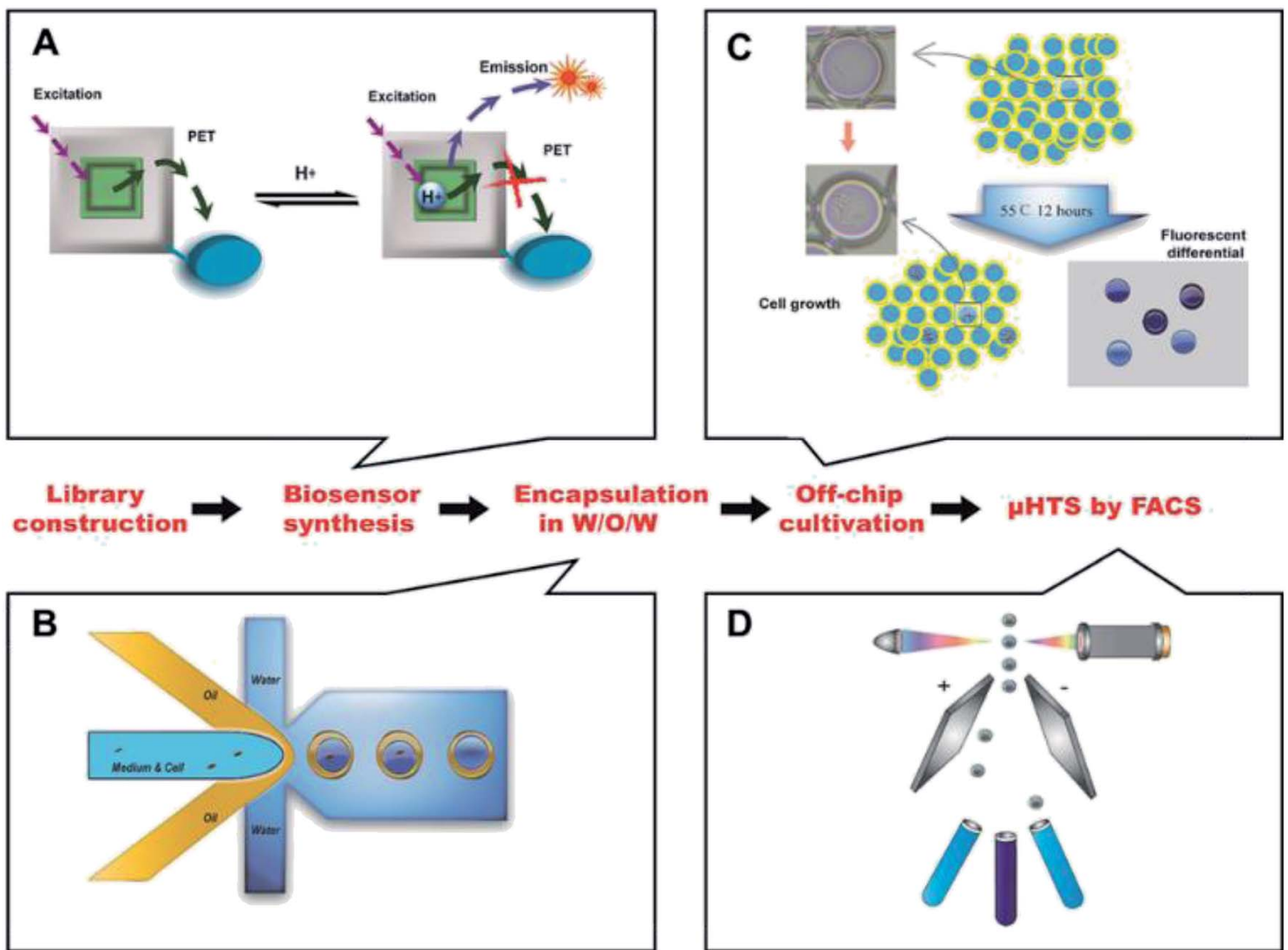

Fig. 1 Schematic illustration of the proposed droplet microfluidic system. (A) The response mechanism of the pH fluorescent biosensor. (B) Design of chip and generation of W/O/W droplets. (C) Cell growing and producing of lactic acid and the response of the fluorescent. (D) Ultrahigh-throughput screening combined with FACS.

\section{Water-in-oil-in-water $(\mathrm{W} / \mathrm{O} / \mathrm{W})$ double emulsion droplets generation}

A one-step $\mathrm{W} / \mathrm{O} / \mathrm{W}$ microdroplets generator was designed and fabricated. The blue print and the dimensions of the chip were shown in Fig. 2A. To produce $\mathrm{W} / \mathrm{O} / \mathrm{W}$ droplets, the surface modification was necessary. ${ }^{27}$ Here, a selective surface modification strategy was put forward. It patterned the wettability of
A
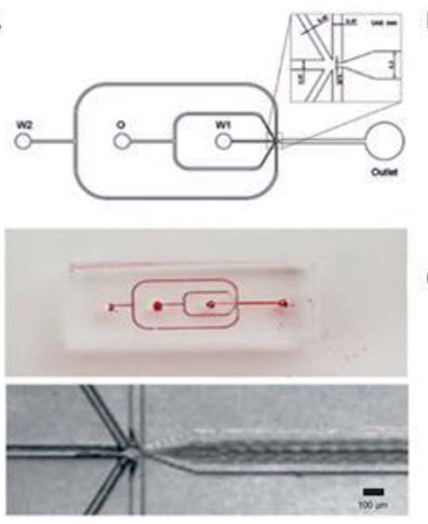

B
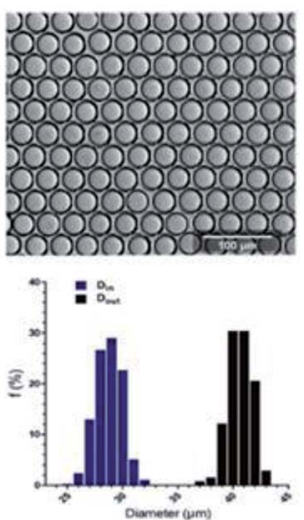

Fig. 2 Chip design and generation of W/O/W microdroplets. (A) Design and photographs of the one-step W/O/W microdroplet generator. Scale bar: $100 \mu \mathrm{m}$. (B) Microphotograph of W/O/W microdroplets. Scale bar: $100 \mu \mathrm{m}$. (C) The outer and inner diameters distribution of W/O/W microdroplets. the device by PEG-200 and Aquapel as described in the experimental section. It was worth noting that the use of a negative pressure system made the patterned surface modifications easier to control and more accurate. Microdroplets generated by this device can produce emulsions with a pretty low variation (Fig. 2B). The inner and outer diameters of more than 1000 droplets were statistics. The diameters distribution was shown in Fig. 2C. The coefficient of variation of the outer and inner diameters were $2.79 \%$ and $4.2 \%$ respectively. This is mainly due to suitable working principle of one-step $\mathrm{W} / \mathrm{O} / \mathrm{W}$ and the decent surface modification. Compared with the two-step $\mathrm{W} / \mathrm{O} / \mathrm{W}$ microdroplet generators the one-step generator worked much better in the production of thin shell W/O/W microdroplets. ${ }^{28,29}$ It can also get a pretty low degree of polydispersity thanks to its droplet generation mechanism: during $\mathrm{W} / \mathrm{O} / \mathrm{W}$ microdroplet generation, the inner aqueous phase (W1) could be surrounded by a sheath of middle phase $(\mathrm{O})$. When the flow rates of the $\mathrm{W} 2$ (the outer aqueous phase) were set to induce a dripping instability, the coaxial jet was pinched into double emulsions.

\section{A high throughput screening model for lactic acid in droplets}

As a commonly used lactic acid-producing bacteria, the mechanism of lactic acid production in Bacillus coagulans has been investigated in-depth. ${ }^{3,30}$ In this process, the produced lactic acid dominated the value of $\mathrm{pH}$ in fermentation broth when no 
neutralizing agent is present. So a screening model using the $\mathrm{pH}$ of the broth to indicate the yield of the lactic acid was convincing. With this idea, we removed neutralizing agents in the medium and the incubation time of Bacillus coagulans in droplets was set at 12 hours.

There were two reasons for the $12 \mathrm{~h}$ cultivation time: firstly, for $12 \mathrm{~h}$ cultivation, the mortality rate of the bacteria was about $95 \%$. This higher mortality rate was beneficial to the screening efficiency of a higher degree of positive mutation. Secondly, for the effectiveness of the screening model, pre-experiments in 96-well plates showed that mutants with higher concentrations of lactic acid at $12 \mathrm{~h}$ could accumulate its advantages. Its yields were higher as well when transferred to a conventional culture mode (lactic acid secreted by the bacteria could be neutralized in time) for $72 \mathrm{~h}$ cultivation. Therefore, as a high-throughput screening model, it is reasonable to set $12 \mathrm{~h}$ cultivation to reduce time costs while ensuring effectiveness. In addition, owing to removing the neutralizing agents, the surviving bacteria showed advantages in resistance to high concentrations of lactic acid because of the screening pressure already existed. Furthermore, we designed and synthesized a novel $\mathrm{pH}$ fluorescence biosensor named Probe1 which was a naphthalimide derivative.

The details of Probe- 1 will be listed in ESI. $\dagger$ The principle of its response was the inhibition of photo-induced electron transfer (PET) when $\mathrm{H}^{+}$existed. As a result, its fluorescence intensity was positive correlation with the lactic acid level in $\mathrm{W} /$ $\mathrm{O} / \mathrm{W}$ droplets. The availability and biocompatibility of Probe-1 was shown in ESI. $\uparrow$ Unlike commercial $\mathrm{pH}$ fluorescent agents, the optimum range of the self-developed $\mathrm{pH}$ fluorescent agent was designed to be $\mathrm{pH} 3$ to 8 , nicely covering the $\mathrm{pH}$ change during the lactic acid fermentation. At the same time, the fluorescence intensity was inversely proportional to the $\mathrm{pH}$ (most commercially available indicators are in a opposite ways), so the aim of FACS was the strongest part of the fluorescence intensity, which made the FACS easier to implement.

To evaluate the performance of the methodology in microdroplets, pre-formed microdroplets using samples taken from the same flask at different time point $(6 \mathrm{~h}, 8 \mathrm{~h}, 10 \mathrm{~h}, 12 \mathrm{~h})$ were observed with a fluorescence imaging system. The DAPI channel of this system was used and all images were taken under the same parameter setting. The $\mathrm{pH}$ of these samples were 4.41, 4.27, $4.21,4.17$, respectively and their lactic acid concentration were $8 \mathrm{~g} \mathrm{~L}^{-1}, 11 \mathrm{~g} \mathrm{~L}^{-1}, 12 \mathrm{~g} \mathrm{~L}^{-1}, 14 \mathrm{~g} \mathrm{~L}^{-1}$. Fig. 3A showed that the fluorescence was increasing with the concentration of lactic acid. Quantitative result was acquired by translating the average fluorescence intensity of each image to the gray value (Fig. 3B). Microdroplets encapsulated with Bacillus coagulans which had been cultured for 10 hours visually displayed different fluorescence as the lactic acid concentration was different (Fig. 3C). These results demonstrated the effectiveness of the fluorescent indicator as well as the feasibility of its application to microdroplets high throughput screening. Successfully converting the lactic acid concentration within the droplet into a fluorescent signal enabled the rapid analysis by a fluorescence detector. That meant we were able to establish a high throughput screening platform with assistance of FACS. For lactic acid detection, assay based on L-lactate oxidase enzyme/horseradish peroxidase/

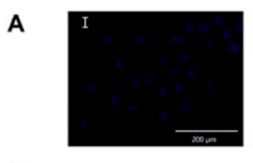

B
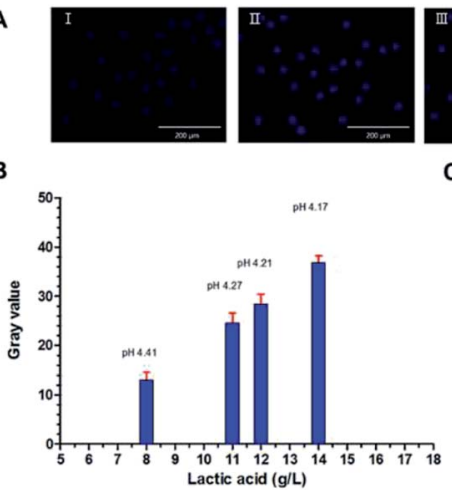

C
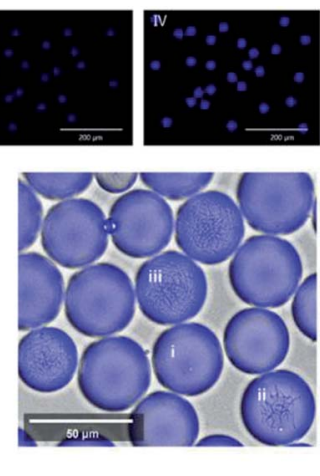

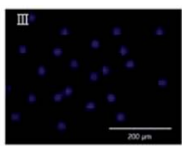

Fig. 3 Lactic acid assay in microdroplets. (A) Fluorescence imaging of microdroplets containing broth with a range of lactic acid, $8 \mathrm{~g} \mathrm{~L}^{-1}$ (I), $11 \mathrm{~g}$ $\mathrm{L}^{-1}$ (II), $12 \mathrm{~g} \mathrm{~L}^{-1}$ (III), $14 \mathrm{~g} \mathrm{~L}^{-1}$ (IV). Scale bar: $200 \mu \mathrm{m}$. (B) The corresponding average gray value derive from the fluorescence intensity of the microdroplets in the images of (A). (C) The microdroplets cultured for 10 hours showed difference in fluorescence. Microdroplets with relative high, low fluorescence intensity and the empty ones were illustrated by (iii), (ii), (i), respectively. Scale bar: $50 \mu \mathrm{m}$.

Amplex UltraRed system was proved validity. In this enzymatic system, the $\mathrm{H}_{2} \mathrm{O}_{2}$ produced from the oxidation of lactic acid could oxidate the Amplex UltraRed to fluorescent resorufin so that the amount of fluorescent resorufin produced was proportional to the concentration of the metabolite of interest in solution (Wang et al. 2014). However, this enzymatic method was unsuitable for off-chip analysis like flow cytometry because it was hard to keep a consistent reaction time in each droplet. The Probe-1 can break this limit because it can indicate the lactic acid in a real-time model. Besides, as a fluorophore reporting for $\mathrm{pH}$ rather than only for lactic acid, the application of the Probe-1 was flexible.

\section{High-throughput sorting and high lactic acid yield strain screening}

Before FACS sorting, a FACS analysis was performed to get valid information and to optimize parameters for sorting. After generation, the $\mathrm{W} / \mathrm{O} / \mathrm{W}$ microdroplets were collected into two sterile EP tube. One was kept in $-4{ }^{\circ} \mathrm{C}$, which was used as control group later and another was cultured for $12 \mathrm{~h}, 55{ }^{\circ} \mathrm{C}$. Then they were going to be analyzed by FACS. Fig. 4A and B showed the Alexa Fluor 430 A against SSC-A (side scatter-area) $\log$-log pseudo-color plots of the sample before and after culture. A quad gate was set based on the pre-analysis of the control group. The Q4 region represented the oil-in-water droplets mixed in the $\mathrm{W} / \mathrm{O} / \mathrm{W}$ droplets, with a ratio of $\sim 15 \%$. They were inevitable due to the slight fluctuation of the liquid flow during the droplet generation. Owing to their lack of fluorescence signals, the presence of these droplets did not affect the results of the sorting, but could be used as a blank for FACS fluorescence analysis. The Q2 + Q3 region represented the W/O/W microdroplets, which showed much stronger fluorescent due to the presence of the Probe-1. After $12 \mathrm{~h}$ cultivation, the growth of Bacillus coagulans and the accumulation of lactic acid in the droplets resulted in a significant increase in fluorescence intensity (the $X$ axis). 


\section{A}

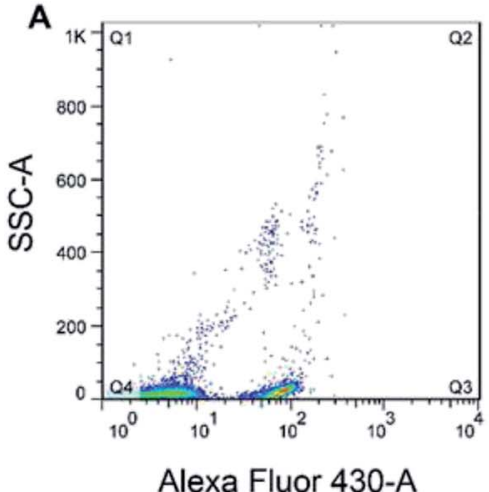

B

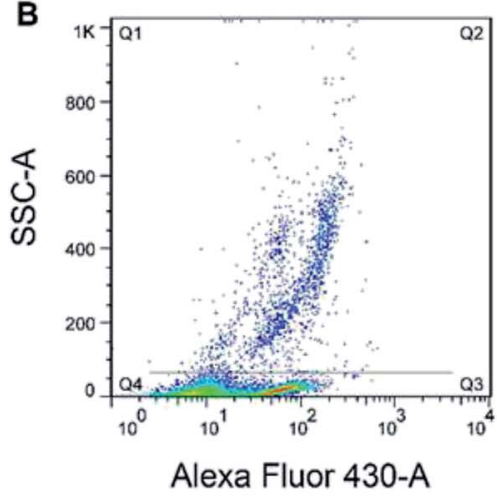

C
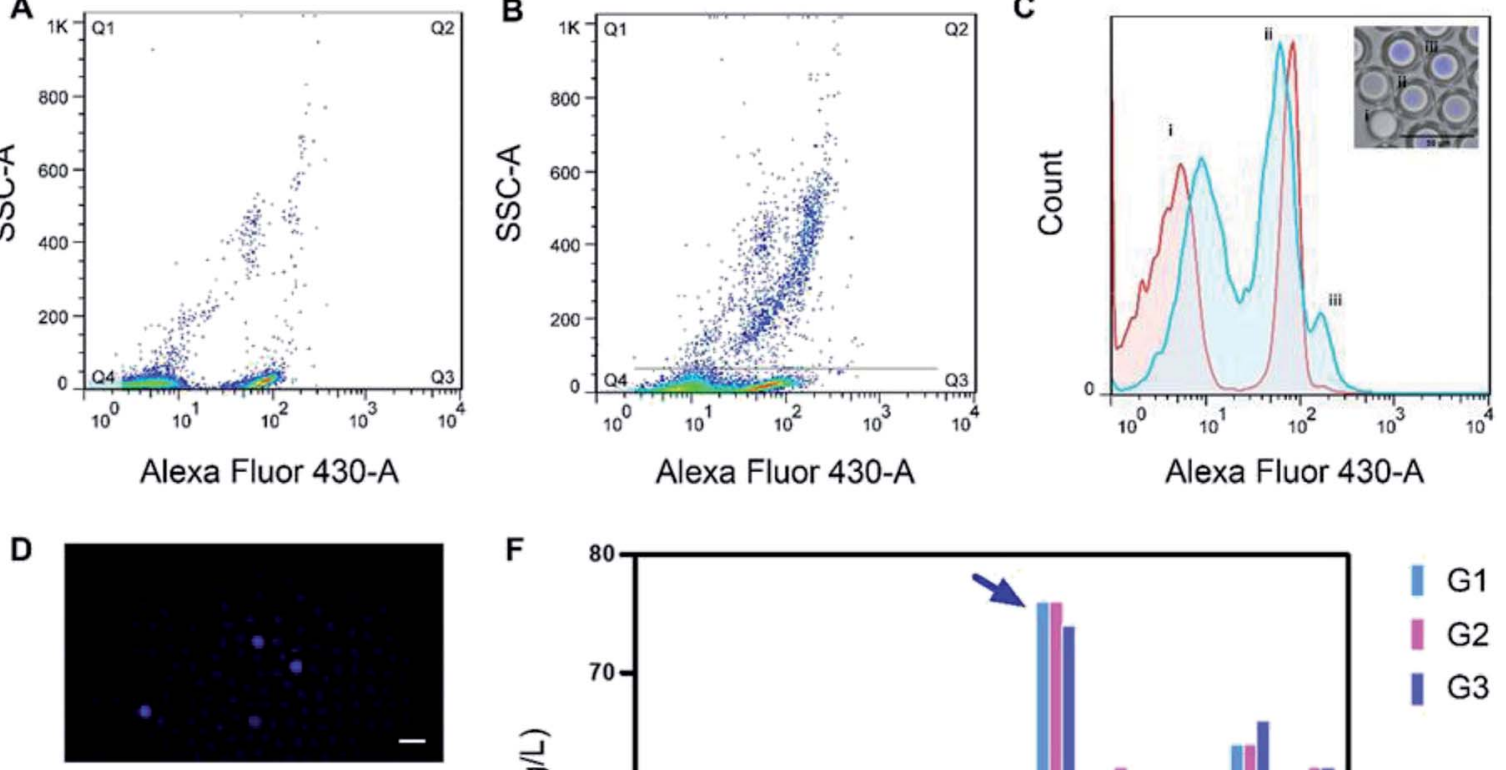

E

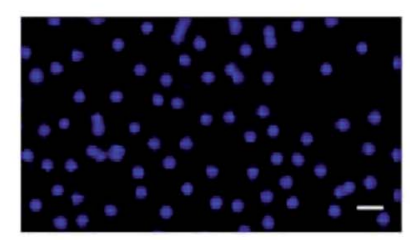

$\mathbf{F}$

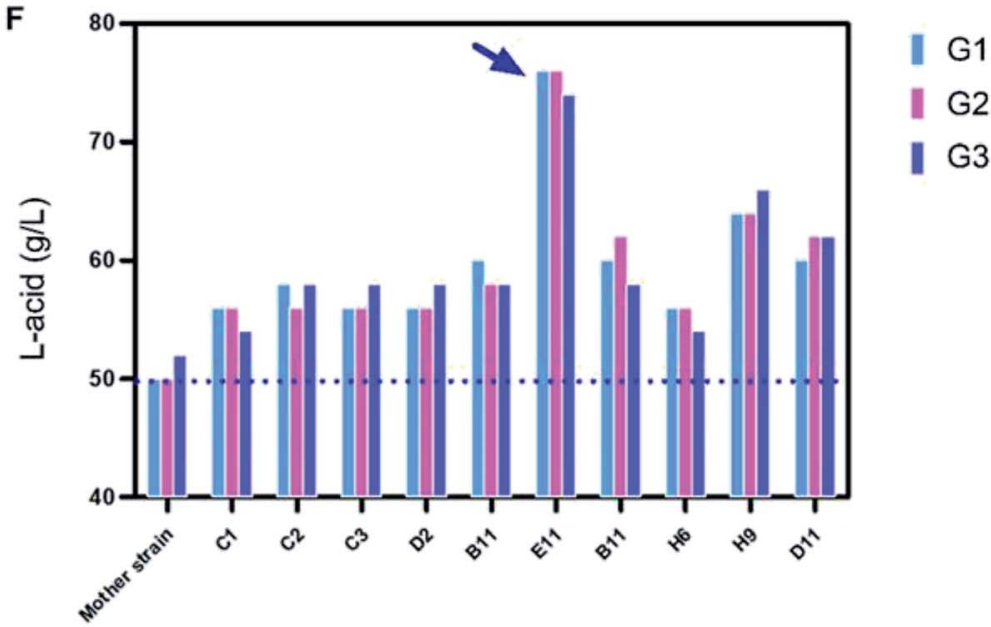

Fig. 4 High throughput screening of W/O/W microdroplets by FACS. (A) Alexa Fluor 430 - A against SSC-A (side scatter-area) log-log plots of the control group, which was not cultured. (B) Alexa Fluor 430 - A against SSC-A (side scatter-area) log-log plots of the samples after cultivation. The Q2 gating events was considered as the microdroplets with cells which were producing lactic acid. (C) Fluorescence frequency histograms derived from all the events of (A) (red) and (B) (blue). Three peaks (i), (ii), (iii) indicated the O/W single emulsion and fragments, W/O/W without cells and W/O/W with cells as shown in the fluorescence images. Scale bar: $50 \mu \mathrm{m}$. (D) and (E) Droplets before and after FACS sorting. It showed an obvious enrichment of droplets with strong fluorescence intensity. Scale bar: $100 \mu \mathrm{m}$ (F) 10 mutants with high yield of lactic acid and three generations hereditary stability was obtained. The labels on the $X$-axis represented the name of 10 mutants, and the three column of each mutant represented the lactic acid production of each generation (G1, G2, G3). The highest yield of mutant E11 reached $76 \mathrm{~g} \mathrm{~L}^{-1}$ (indicated by blue arrow), $52 \%$ higher than its mother strains.

This showed an increase of frequency in Q2 from $2.08 \%$ to $14.4 \%$ as shown in Fig. 4B. Besides, the growing and division of Bacillus coagulans showed an increase of side scatter (the $Y$ axis). Histogram Fig. 4C was corresponding to Fig. 4A (the red histogram) and Fig. 4B (the blue histogram). It clearly showed their difference in the fluorescent signal before and after culture. Three peaks (i), (ii), (iii) indicated the single $\mathrm{O} / \mathrm{W}$ emulsion and fragments, $\mathrm{W} / \mathrm{O} / \mathrm{W}$ without cells and $\mathrm{W} / \mathrm{O} / \mathrm{W}$ with cells. They were in accordance with the fluorescence images of the microdroplets cultured for $12 \mathrm{~h}, 55{ }^{\circ} \mathrm{C}$.

For screening, W/O/W microdroplets with mutant candidates inside was incubated $12 \mathrm{~h}$ at $55{ }^{\circ} \mathrm{C}$ to produce lactic acid. Then, the library was screened by FACS with a sort gate set to the highest $5 \%$ of Alexa Fluor 430 - A events. Since the mortality rate in the droplets exceeds $95 \%$ after cultivation, we have to use such a high cut-off. We tried lower cut-offs ( $1 \%$ and $3 \%)$, but it showed that the cell viability of collected cells was 0 . After one round sorting, approximately $1 \times 10^{6}$ droplets were interrogated and collected in one hour. The fluorescence micrograph of the droplets before (Fig. 4D) and after (Fig. 4E) FACS showed an obvious enrichment of droplets with strong fluorescence intensity. These results show the effectiveness of our screening system. Here, taking advantage of droplet-based microfluidics and flow cytometry and we built a high throughput screening system for high lactic acid-producing Bacillus coagulans. This system realized high throughput screening for extracellular metabolites on single cell level by encapsulating one single cell in the droplet. Its screening throughput exceeded $1 \times 10^{6}$ per hour. Usually, using a microplate reader to analyze a 96-well plate takes about 5 minutes. Under this case, the screening throughput was more than 1000 times higher than the customary 96-well-plate. In terms of material consumption, micro-droplet technology was 7 to 8 orders of magnitude lower than microplate technology.

\section{Verification of high-yield lactic acid mutans}

These sorted candidates were further screened by 96-well-plate and the top 100 mutants were validated in flasks. Finally, 10 mutants with increasing in the yield of lactic acid and three 
generations hereditary stability were obtained. The highest yield of mutant E11 reached $76 \mathrm{~g} \mathrm{~L}^{-1}, 52 \%$ higher than the mother strain (Fig. 4F). $76 \mathrm{~g} \mathrm{~L}^{-1}$ was also a quite high level of lactic acid yield for flask culture.

\section{Experimental}

\section{Strains and media}

Bacillus coagulans HL5-C, a derivative of the industrial strain $B$. coagulans (CGMCC no. 1.2407) obtained by random mutagenesis was used for L-lactic acid production. The liquid screening medium contains $90 \mathrm{~g} \mathrm{~L}^{-1}$ glucose, $13.33 \mathrm{~g} \mathrm{~L}^{-1}$ yeast extract, $13.33 \mathrm{~g} \mathrm{~L}^{-1}$ tryptone, $0.67 \mathrm{~g} \mathrm{~L}^{-1} \mathrm{CH}_{3} \mathrm{COONa}, 0.01 \mathrm{~g} \mathrm{~L}^{-1} \mathrm{NaCl}$, $0.01 \mathrm{~g} \mathrm{~L}^{-1} \mathrm{MgSO}_{4}, 0.01 \mathrm{~g} \mathrm{~L}^{-1} \mathrm{FeSO}_{4}, 0.01 \mathrm{~g} \mathrm{~L}^{-1} \mathrm{MnSO}_{4}$, and $10^{-5} \mathrm{~mol} \mathrm{~L}^{-1}$ Probe-1 (the homemade fluorescent reagent). Medium for seed culture in flask contains $40 \mathrm{~g} \mathrm{~L}^{-1}$ glucose, $10 \mathrm{~g}$ $\mathrm{L}^{-1}$ yeast extract, $10 \mathrm{~g} \mathrm{~L}^{-1}$ beef extract, $10 \mathrm{~g} \mathrm{~L}^{-1}$ tryptone, $4 \mathrm{~g} \mathrm{~L}^{-1}$ $\mathrm{CH}_{3}$ COONa, $2 \mathrm{~g} \mathrm{~L}^{-1}$ diammonium citrate, $2 \mathrm{~g} \mathrm{~L}^{-1} \mathrm{~K}_{2} \mathrm{HPO}_{4}$, $0.03 \mathrm{~g} \mathrm{~L}^{-1} \mathrm{NaCl}, 0.02 \mathrm{~g} \mathrm{~L}^{-1} \mathrm{MgSO}_{4}, 0.02 \mathrm{~g} \mathrm{~L}^{-1} \mathrm{MnSO}_{4}, 0.01 \mathrm{~g} \mathrm{~L}^{-1}$ $\mathrm{FeSO}_{4}$ and $25 \mathrm{~g} \mathrm{~L}^{-1} \mathrm{CaCO}_{3}$. The flask fermentation medium was the liquid screening medium with extra $40 \mathrm{~g} \mathrm{~L}^{-1} \mathrm{CaCO}_{3}$. All mediums were adjusted to $\mathrm{pH} 6.0$ with $\mathrm{HCl}$ or $\mathrm{NaOH}$, and sterilized for $20 \mathrm{~min}$ at $115{ }^{\circ} \mathrm{C}$.

\section{Culture conditions}

For droplets, after generation, the microdroplets were collected in a $2 \mathrm{~mL}$ EP tube and cultured at $55^{\circ} \mathrm{C}$ for $12 \mathrm{~h}$ in the incubator. For 96-well MTPs, the mutants were inoculated in 96-well MTPs with $100 \mu \mathrm{L}$ liquid screening medium in each well at $200 \mathrm{rpm}$ shaking for $12 \mathrm{~h}$. For shake flasks, the method was detailed in our previous work. ${ }^{31}$

\section{Construction of mutagenesis library}

The combination of Atmospheric and Room Temperature Plasma (ARTP) and sodium nitrite was used to construct the mutagenesis library. During the exponential growth phase, the bacteria suspension was taken and diluted with saline to $\mathrm{OD}_{620}$ $=2.0\left(\sim 10^{8}\right.$ cells per $\left.\mathrm{mL}\right)$. The bacteria suspension was firstly treated with sodium nitrite. $1 \mathrm{~mL}$ of bacteria suspension mixed with $1 \mathrm{~mL}$ sodium nitrite solution $(0.1 \mathrm{M})$ and $2 \mathrm{~mL}$ acetate buffer (0.1 M, pH 4.5). After incubation at $28^{\circ} \mathrm{C}$ for $90 \mathrm{~s}, 20 \mathrm{~mL}$ $\mathrm{Na}_{2} \mathrm{HPO}_{4}(0.7 \mathrm{M}, \mathrm{pH}$ 8.6) was added to terminate the effect of sodium nitrite at $\mathrm{pH}$ 7.0. Then $10 \mu \mathrm{L}$ of the suspension was dipped onto the sterilized metal plate $(5 \mathrm{~mm}$ diam.) and was treated with the atmospheric and room temperature plasma (ARTP Mutagenesis Breeding Machine, CHN) for $20 \mathrm{~s}$ at a nitrogen flow rate of $10 \mathrm{~L} \mathrm{~min} \min ^{-1}$ and $10 \mathrm{~mm}$ irradiation distance. Candidate mutants from 10 metal plates were washed with $9900 \mu \mathrm{L}$ sterilized seed medium and then cultured at $55{ }^{\circ} \mathrm{C}$ till the $\mathrm{OD}_{620}$ reached $1.0\left(\sim 3.5 \times 10^{6}\right.$ cells per $\left.\mathrm{mL}\right)$.

\section{L-Lactic acid detection}

A lactic acid detector SBA-40E (Shandong Province Academy of Sciences, Shandong, China $)^{31}$ was used to specifically determine the L-lactic acid in the fermentation broth using a built-in immobilized L-lactic acid oxidase reaction system. The L-lactic acid diluted to a certain concentration range $\left(0-100 \mathrm{mg} \mathrm{dL} \mathrm{dL}^{-1}\right)$ could be quickly and precisely analyzed.

\section{Microfluidic system fabrications and surface modification}

The pattern of microfluidics device was designed by AutoCAD, and then transferred to a high-resolution plotted film, which was bonded to a customized quartz glass resulting to a compositional photo mask. The negative photoresist SU-8 2075 (MicroChem, USA) was spin-coated onto a 4-inch silicon wafer. A mask aligner was subsequently used for photoetching and lead to a male mold in the silicon wafer. PDMS prepolymer (Dow Corning, USA) and curing agent with a ratio $10: 1$ were mixed and violently stirred. PDMS mixture was poured onto the silicon master and degassed in a vacuum oven, then cured at $65{ }^{\circ} \mathrm{C}$ overnight. After curing, the PDMS replica containing microchannel pattern was peeled away from the silicon master. A hole puncher was used to make holes in set position in order to form the inlets and outlets for the samples and reagents. The PDMS replica and glass slides were exposed to an oxygen plasma (Harrick Plasma, USA) for $180 \mathrm{~s}$ at 300 watts, and then face-to-face bonded. The dimensions of the microfluidics device were $100 \mu \mathrm{m}$ for branch channels and $200 \mu \mathrm{m}$ for main channels, while the angle of these two branches was $60^{\circ}$.

Surface treatment of the microfluidic channels was needed to generate double emulsion droplets. Patterning of the wettability was accomplished to generate $\mathrm{W} / \mathrm{O} / \mathrm{W}$ (water in oil in water) microdroplets. ${ }^{32}$ A straightforward method for generating stable hydrophilic coatings with poly-(ethylene glycol)-200 (PEG-200) (Macklin, INC, CN) ${ }^{33}$ was improved and a method based on negative pressure drainage was constructed to make patterned hydrophilic and hydrophobic modification. In brief, after plasma bonding the PDMS replica to the slide, PEG-200 was immediately drawn in the channel from the outlet by vacuum aspiration at the inlet W2. The device was placed onto a hot plate at $150{ }^{\circ} \mathrm{C}$ for $30 \mathrm{~min}$. During heating, the vacuum aspiration still worked and the PEG-200 was continuously supplemented. Then, the device was washed with isopropanol and water to remove residual PEG-200. In the second step, a commercial water repellent agent Aquapel (Pittsburgh Glass Works, LLC, USA) was used to produce a defined hydrophobic surface in the microchannel. The vacuum aspiration was used at the inlet W2. The Aquapel was drawn in from the inlet W1 and inlet $\mathrm{O}$ for $10 \mathrm{~min}$.

\section{Double emulsion droplet generation and detection}

For $\mathrm{W} / \mathrm{O} / \mathrm{W}$ microdroplets, the inner phase was the liquid screening medium with bacteria and fluorescein inside. QX200 ${ }^{\mathrm{TM}}$ Droplet Generation Oil-EvaGreen (Bio-Rad Laboratories, INC, USA) was used as the middle phase. For the outer phase, we used deionized water with $10 \%(\mathrm{w} / \mathrm{w})$ Tween-80 and $10 \%$ glycerin $(\mathrm{w} / \mathrm{w})$ as the surfactant. The flow rates of the medium, oil and the outer aqueous phase were set at 40,80 and $1500 \mu \mathrm{L} \mathrm{h}^{-1}$ respectively. All solutions were delivered into the microfluidic channels using 1 or $5 \mathrm{~mL}$ syringes (BD Plastipak syringes, VWR, UK) and precision syringe pumps. 


\section{Microscopic imaging of droplets}

EVOS FL Auto Imaging System (Thermo Fisher Scientific, USA) was used for the microscopic imaging of the droplets. For bright-field microscopic images, droplets were distributed on the glass slide and were observed under $400 \times$ magnification. For fluorescent microscopic images, in order to minimize the impact of background fluorescence of the medium, the DAPI channel (Ex: 357/44 nm, Em: 447/60 nm) was chosen rather than the GFP (Ex: 470/22 nm, Em: 510/42 nm) channel even though the latter had a better match with the max Ex/Em wavelength of the fluorescein Probe-1 (Ex/Em: 405 nm/530 nm).

\section{Flow cytometry analysis}

FACS Aria (BD Biosciences, USA) was used to conduct the FACS analysis. Sterile PBS was used as sheath fluid and the sorter was equipped with a $100 \mu \mathrm{m}$ nozzle. The analysis rate was 500-1000 events per s. Laser of $405 \mathrm{~nm}$ was used for excitation of the fluorescent biosensor and the emission passed through a $530 \pm$ $30 \mathrm{~nm}$ band-pass filter (Alexa Fluor 430 channel), which matched with the max Ex/Em of the homemade fluorescein Probe-1. For analyzing, the $\mathrm{W} / \mathrm{O} / \mathrm{W}$ microdroplets stained with fluorescein were diluted with $20 \%(\mathrm{w} / \mathrm{w})$ glucose solution in a $1: 1$ ratio $(\mathrm{v} / \mathrm{v})$ after collection to balance the osmotic pressure.

\section{High-throughput microdroplets sorting with flow cytometry}

The mutant library was screened by FACS using a sort gate set to the highest $5 \%$ of Alexa Fluor 430 - A events. The sorting rate was 100-500 events per s. The sorted drops were collected in water containing $10 \%(\mathrm{w} / \mathrm{w})$ Tween-80, 10\% (w/w) glycerin and $10 \%(\mathrm{w} / \mathrm{w})$ glucose (to balance osmotic pressure).

\section{Rapid screening using 96-well-plate}

The emulsion of drops sorted by FACS was removed. The broth was evenly divided into 96-well-plates with Probe-1. 96-wellplates were loaded with $100 \mu \mathrm{L}$ medium which contained $10^{-5} \mathrm{~mol} \mathrm{~L}^{-1}$ Probe- 1 and inoculated with candidate mutants for $12 \mathrm{~h}$ at $55{ }^{\circ} \mathrm{C}, 200 \mathrm{rpm}$ cultivation. And directly detected by microplate reader for fluorescence intensity with Ex/Em: 405 $\mathrm{nm} / 530 \mathrm{~nm}$. High-yield strain was isolated from the high-yield well as described in our previous work (Lv et al. 2016a).

\section{Conclusions}

In this work, we described a high throughput screening system for lactic acid-producing bacteria by which cells are encapsulated in $\mathrm{W} / \mathrm{O} / \mathrm{W}$ droplets. A fluorescent reporter was used to determine $\mathrm{pH}$ changes in the droplet. Droplets were then sorted using FACS. We designed a one-step W/O/W microdroplet generator. A screening model for lactic acid in droplets was built. At the same time, after a round of actual screening, the effectiveness of the system was verified. This system was valuable with its ultrahigh throughput and little reagent consumption (pico-liter level). With the assistance of microfluidics, the application of the FACS technique can be broadened to the field of extracellular metabolites sorting. Except detection of the production of lactic acid, the growing and division of Bacillus coagulans can also be detected by the side scatter channel of FACS at the same time. This feature allowed further study of the productivity of typical strains.

Furthermore, the accessible feature of the system will also enable the screening process of other microbes with slight modifications, provided that the metabolites can be linked to a fluorescent signal.

\section{Conflicts of interest}

There are no conflicts to declare.

\section{Acknowledgements}

This study was financially supported by the National Science Foundation for Young Scientists of China (31700038), the National Natural Science Foundation of China (21878084) and the National Key Research and Development Program (2017YFB0309302).

\section{Notes and references}

1 F. A. C. Martinez, E. M. Balciunas, J. M. Salgado, J. M. D. Gonzalez, A. Converti and R. P. D. Oliveira, Trends Food Sci. Technol., 2013, 30, 70-83.

2 R. Datta and M. Henry, J. Chem. Technol. Biotechnol., 2006, 81, 1119-1129.

3 M. A. Eiteman and S. Ramalingam, Biotechnol. Lett., 2015, 37, 955-972.

4 N. Narayanan, P. K. Roychoudhury and A. Srivastava, Electron. J. Biotechnol., 2004, 7, 167-179.

5 R. P. John, K. M. Nampoothiri and A. Pandey, Appl. Microbiol. Biotechnol., 2007, 74, 524-534.

6 A. Savadogo, C. A. T. Ouattara, I. H. N. Bassole and S. A. Alfred, Afr. J. Biotechnol., 2006, 5, 678-683.

7 S. K. Singh, S. U. Ahmed and A. Pandey, Process Biochem., 2006, 41, 991-1000.

8 S. Michael, D. Auld, C. Klumpp, A. Jadhav, W. Zheng, N. Thorne, C. P. Austin, J. Inglese and A. Simeonov, Assay Drug Dev. Technol., 2008, 6, 637-657.

9 W. F. An and N. Tolliday, Mol. Biotechnol., 2010, 45, 180-186. 10 M. Entzeroth, Curr. Opin. Pharmacol., 2003, 3, 522-529.

11 Q. Long, X. Liu, Y. Yang, L. Li, L. Harvey, B. McNeil and Z. Bai, J. Biotechnol., 2014, 192(pt B), 323-338.

12 L. M. Mayr and P. Fuerst, J. Biomol. Screening, 2008, 13, 443448.

13 L. M. Mayr and D. Bojanic, Curr. Opin. Pharmacol., 2009, 9, 580-588.

14 L. Wu, P. Chen, Y. Dong, X. Feng and B.-F. Liu, Biomed. Microdevices, 2013, 15, 553-560.

15 P.-Y. Colin, A. Zinchenko and F. Hollfelder, Curr. Opin. Struct. Biol., 2015, 33, 42-51.

16 A. Autour and M. Ryckelynck, Micromachines, 2017, 8, 128.

17 P. Mair, F. Gielen and F. Hollfelder, Curr. Opin. Chem. Biol., 2017, 37, 137-144. 
$18 \mathrm{X}$. Sun, W. D. Weinlandt, H. Patel, M. Wu and C. J. Hernandez, Lab Chip, 2014, 14, 2491-2498.

19 K. Eyer, P. Kuhn, S. Stratz and P. S. Dittrich, J. Visualized Exp., 2013, 80, 50618.

20 A. J. Hughes and A. E. Herr, Proc. Natl. Acad. Sci. U. S. A., 2012, 109, 21450-21455.

21 S. A. Vanapalli, M. H. Duits and F. Mugele, Biomicrofluidics, 2009, 3, 12006.

22 F. V. Ignatovich, D. Topham and L. Novotny, IEEE J. Sel. Top. Quantum Electron., 2006, 12, 1292-1300.

23 E. K. Sackmann, A. L. Fulton and D. J. Beebe, Nature, 2014, 507, 181-189.

24 R. Ostafe, R. Prodanovic, J. Nazor and R. Fischer, Chem. Biol., 2014, 21, 414-421.

25 J. Yan, W. A. C. Bauer, M. Fischlechner, F. Hollfelder, C. F. Kaminski and W. T. S. Huck, Micromachines, 2013, 4, 402-413.

26 J. Fattaccioli, J. Baudry, J. D. Emerard, E. Bertrand, C. Goubault, N. Henry and J. Bibette, Soft Matter, 2009, 5, 2232-2238.
27 W. A. Bauer, M. Fischlechner, C. Abell and W. T. Huck, Lab Chip, 2010, 10, 1814-1819.

28 J. M. Morais, O. D. H. Santos and S. E. Friberg, J. Dispersion Sci. Technol., 2010, 31, 1019-1026.

29 A. R. Abate, J. Thiele and D. A. Weitz, Lab Chip, 2011, 11, 253258.

30 X. Y. Lv, B. Yu, X. W. Tian, Y. Chen, Z. J. Wang, Y. P. Zhuang and Y. H. Wang, J. Taiwan Inst. Chem. Eng., 2016, 61, 124131.

31 X. Lv, J. Song, B. Yu, H. Liu, C. Li, Y. Zhuang and Y. Wang, Bioprocess Biosyst. Eng., 2016, 39, 1737-1747.

32 M. Lee, K. H. Kim, J. G. Park, J. H. Lee, H. W. Lim, M. Y. Park, S. I. Chang, E. K. Lee, D. W. Lim and J. Choo, BioChip J., 2012, 6, 10-16.

33 P. B. Lillehoj and C.-M. Ho, A Long-term, Stable Hydrophilic Poly(dimethylsiloxane) Coating for Capillary-Based Pumping, Proceedings of the 2010 IEEE 23rd International Conference on Micro Electro Mechanical Systems (MEMS), Wanchai, Hong Kong, pp. 1063-1066. 